\title{
Review Article \\ The Role of miR-378a in Metabolism, Angiogenesis, and Muscle Biology
}

\author{
Bart Krist, Urszula Florczyk, Katarzyna Pietraszek-Gremplewicz, \\ Alicja Józkowicz, and Jozef Dulak
}

Department of Medical Biotechnology, Faculty of Biochemistry, Biophysics and Biotechnology, Jagiellonian University, Gronostajowa 7, 30-387 Krakow, Poland

Correspondence should be addressed to Jozef Dulak; jozef.dulak@uj.edu.pl

Received 15 October 2015; Accepted 30 November 2015

Academic Editor: Young-Ah Moon

Copyright (C) 2015 Bart Krist et al. This is an open access article distributed under the Creative Commons Attribution License, which permits unrestricted use, distribution, and reproduction in any medium, provided the original work is properly cited.

\begin{abstract}
MicroRNA-378a (miR-378a, previously known as miR-378) is one of the small noncoding RNA molecules able to regulate gene expression at posttranscriptional level. Its two mature strands, miR-378a-3p and miR-378a-5p, originate from the first intron of the peroxisome proliferator-activated receptor gamma, coactivator 1 beta ( $p$ pargclb) gene encoding PGC- $1 \beta$. Embedding in the sequence of this transcriptional regulator of oxidative energy metabolism implies involvement of miR-378a in metabolic pathways, mitochondrial energy homeostasis, and related biological processes such as muscle development, differentiation, and regeneration. On the other hand, modulating the expression of proangiogenic factors such as vascular endothelial growth factor, angiopoietin1, or interleukin-8, influencing inflammatory reaction, and affecting tumor suppressors, such as SuFu and Fus-1, miR-378a is considered as a part of an angiogenic network in tumors. In the latter, miR-378a can evoke broader actions by enhancing cell survival, reducing apoptosis, and promoting cell migration and invasion. This review describes the current knowledge on miR378a linking oxidative/lipid metabolism, muscle biology, and blood vessel formation.
\end{abstract}

\section{Introduction}

Cell metabolism governing the growth and functioning of each cell and a whole organism refers to chemical transformations and enzyme-catalyzed energy producing and energy utilizing reactions of carbohydrates, proteins, and lipids. Amongst the most metabolically active organs are liver, brain, gut, kidneys, and heart [1-3]. Although the rate of metabolic reactions is lower in skeletal muscles, they account for around $20 \%$ of the total energy expenditure due to a $50-60 \%$ contribution to a total body mass [3]. Several microRNAs were reported to control processes related to metabolism such as insulin secretion (miR-9, miR-375), adipocyte differentiation (miR-143), fatty acid metabolism (miR-122), and myogenesis (miR-1, miR-133a, miR-133b, and miR-206) (reviewed in [4]). Of potential meaning is also miR-378a, located in the gene encoding master metabolic regulator, peroxisome proliferator-activated receptor gamma, coactivator 1 beta $(\mathrm{PGC}-1 \beta)$ [5]. miR-378a was found to affect lipid and xenobiotic metabolism, lipid storage, mitochondrial function, and shift towards a glycolytic pathway (Warburg effect) $[5,6]$.
Moreover, it affects muscle differentiation via regulation of myogenic repressor, MyoR [7]. Because nutrients supply for metabolic processes is a matter of circulation, metabolically active tissues require high vascular density. Recently, miR378 a was reported to regulate tumor angiogenesis mainly via inhibition of tumor suppressors SuFu and Fus-1 $[8,9]$. Thus, a growing body of evidence suggests a role of miR-378a as a mediator controlling reciprocally dependent processes such as metabolism, muscle differentiation/regeneration, and angiogenesis.

\section{MicroRNAs}

MicroRNAs (miRNAs; miRs) are small noncoding RNA molecules with an average length of 21-22 nucleotides which can regulate gene expression posttranscriptionally by targeting mostly the $3^{\prime}$ untranslated region ( $\left.3^{\prime} \mathrm{UTR}\right)$ of mRNAs. However, miRNA target sites were also found on the $5^{\prime}$ UTR regions of human mRNA [10]. Since their discovery in $C$. elegans in 1993 [11], miRNAs currently can be recognized as potent players in wide spectrum of biological processes like 
TABLE 1: Classification of miR-378 variants. Source: miRBase, version 21, September 2015 [28]. The seed sequence (defined as nucleotides 2-8 from the miRNA $5^{\prime}$-end of the mature miRNA) is in bold font.

\begin{tabular}{|c|c|c|c|c|c|}
\hline Name & Mature strand & Previous ID & Sequence & Location & Host gene \\
\hline \multicolumn{6}{|c|}{ Human } \\
\hline \multirow[b]{2}{*}{ hsa-miR-378a } & hsa-miR-378a-5p & $\operatorname{miR}-378^{*}$ & $5^{\prime}$-cuccugacuccagguccugugu- $3^{\prime}$ & chr5: $149732825-149732890$ & PPARGC1B \\
\hline & hsa-miR-378a-3p & \multirow[t]{11}{*}{$\begin{array}{c}\operatorname{miR}-422 b \\
\operatorname{miR}-378\end{array}$} & $5^{\prime}$-acuggacuuggagucagaaggc $-3^{\prime}$ & chr5: 149732825-149732890 & PPARGC1B \\
\hline hsa-miR-378b & hsa-miR-378b & & $5^{\prime}$-acuggacuuggaggcagaa- $3^{\prime}$ & chr3: 10330229-10330285 & $A T P 2 B 2$ \\
\hline hsa-miR-378c & hsa-miR-378c & & $5^{\prime}$-acuggacuuggagucagaagagugg- $3^{\prime}$ & chr10: $130962588-130962668$ & - \\
\hline hsa-miR-378d-1 & hsa-miR-378d & & $5^{\prime}$-acuggacuuggagucagaaa- $3^{\prime}$ & chr4: 5923275-5923328 & - \\
\hline hsa-miR-378d-2 & hsa-miR-378d & & $5^{\prime}$-acuggacuuggagucagaaa- $3^{\prime}$ & chr8: 93916022-93916119 & PDP1 \\
\hline hsa-miR-378e & hsa-miR-378e & & $5^{\prime}$-acuggacuuggagucagga- $3^{\prime}$ & chr5: 170028488-170028566 & DOCK2 \\
\hline hsa-miR-378f & hsa-miR-378f & & $5^{\prime}$-acuggacuuggagccagaag $-3^{\prime}$ & chr1: 23929070-23929147 & - \\
\hline hsa-miR-378g & hsa-miR-378g & & $5^{\prime}$-acugggcuuggagucagaag- $3^{\prime}$ & chr1: 94745860-94745900 & LINC01057 \\
\hline hsa-miR-378h & hsa-miR-378h & & $5^{\prime}$-acuggacuuggugucagaugg- $3^{\prime}$ & chr5: 154829458-154829540 & FAXDC2 \\
\hline hsa-miR-378i & hsa-miR-378i & & $5^{\prime}$-acuggacuaggagucagaagg- $3^{\prime}$ & chr22: 41923222-41923297 & TNFRSF13C \\
\hline hsa-miR-378j & hsa-miR-378j & & $5^{\prime}$-acuggauuuggagccagaa-3' & chr17: 37614931-37615039 & DDX52 \\
\hline \multicolumn{6}{|c|}{ Murine } \\
\hline \multirow{2}{*}{ mmu-miR-378a } & mmu-miR-378a-5p & $\operatorname{miR}-378^{*}$ & $5^{\prime}$-cuccugacuccagguccugugu- $3^{\prime}$ & chr18: 61397835-61397900 & PPARGC1B \\
\hline & mmu-miR-378a-3p & miR-378 & $5^{\prime}$-acuggacuuggagucagaagg- $3^{\prime}$ & chr18: 61397835-61397900 & PPARGC1B \\
\hline mmu-miR-378b & mmu-miR-378b & & $5^{\prime}$-cuggacuuggagucagaaga- $3^{\prime}$ & chr11: $88352773-88352864$ & MSI2 \\
\hline mmu-miR-378c & mmu-miR-378c & & $5^{\prime}$-acuggacuuggagucagaagc- $3^{\prime}$ & chr14: 46954830-46954928 & SAMD4 \\
\hline mmu-miR-378d & mmu-miR-378d & & $5^{\prime}$-acuggccuuggagucagaaggu- $3^{\prime}$ & chr10: $126710282-126710391$ & - \\
\hline
\end{tabular}

The “*” sign refers to a nucleotide position not present in the murine and rat miR-378a-3p mature sequence, which is present in the mature human sequence.

development, differentiation, cellular defense mechanisms, and others. Conservative estimates state that over $30 \%$ of mRNA expression is regulated by miRNAs [12, 13]. However, others suggest that even up to $60 \%$ of the mRNA expression is targeted by miRNAs [14]. miRNAs are often located in the introns of coding genes or noncoding sequences but can also be located in exons. Intronic miRNAs can be expressed together with their host gene mRNA being derived from a common RNA transcript $[15,16]$. Other miRNAs can also have their own promoters, which enable independent expression, or can be organized in clusters sharing a common transcriptional regulation $[17,18]$.

miRNAs transcription is RNA polymerase II-dependent [17]. In the case of miRNAs that are encoded in their own genes, the primary miRNA transcript (pri-miRNA) is several kilobases long, while miRNAs encoded in intronic regions of other genes (miRtrons) have shorter transcripts. The miRNA stem loop is excised from pri-miRNA by endoribonuclease drosha/DGCR8 (microprocessor complex) and a hairpin called pre-miRNA is exported from the nucleus by exportin- 5 in a Ran-GTP dependent manner [19]. An endoribonuclease dicer removes the hairpin loop sequence from pre-miRNA, creating a double stranded miRNA duplex. Depending on the relative stability of the miRNA duplex, one or, more rarely, both strands can be incorporated in a multiprotein RNAinduced silencing complex (RISC). When there is perfect pairing between the miRNA sequence and its target site, mRNA is cleaved by a protein part of the RISC called argonaute (AGO). If the pairing is partial, deadenylation of the mRNA via recruitment of the CCR4-NOT complex by the GW182 proteins inside the RISC takes place and the poly-A tail is lost, leaving the mRNA vulnerable to RNase activity, ubiquitination, and mRNA degradation. Alternatively, miRNA-induced RISC can also cause repression of translation by mechanisms such as, for example, the promotion of ribosome drop-off from the mRNA transcript or destabilization of the mRNA binding cap protein (Figure 1) (reviewed in $[20,21]$ ).

\section{3. miR-378a: Basics}

miR-378a is embedded in the first intron of the ppargclb gene encoding PGC- $1 \beta[5]$. The pre-miR gives rise to a leading strand (miR-378a-3p, previous IDs for murine sequence: mmu-miR-422b, mmu-miR-378, and mmu-miR-378-3p; for human: hsa-miR-422b and hsa-miR-378) and a passenger strand (miR-378a-5p, previous IDs for murine sequence: mmu-miR-378, mmu-miR-378* , and mmu-miR-378-5p; for human: hsa-miR-378 and hsa-miR-378*). miRNA-378a-3p mature strand was first identified in 2004 in humans (originally named miR-422b) [22]. Recently, other miRs with similar sequences but other localizations in the genome have been discovered and named: mmu-miR-378b,c,d in mouse and hsa-miR-378-b,c,d1,d2,e,f,g,h,i,j in human [23-27] (Table 1). In humans, miR-378a is by far the most expressed of the miR-378 sequences, with 7030 reads per million, in 78 experiments during deep sequencing, compared with 1013220 reads per million, in 42-72 experiments for the other forms, respectively. In mice, miR-378a and miR-378b have similar expression levels, at 11700 and 11000 reads per million 


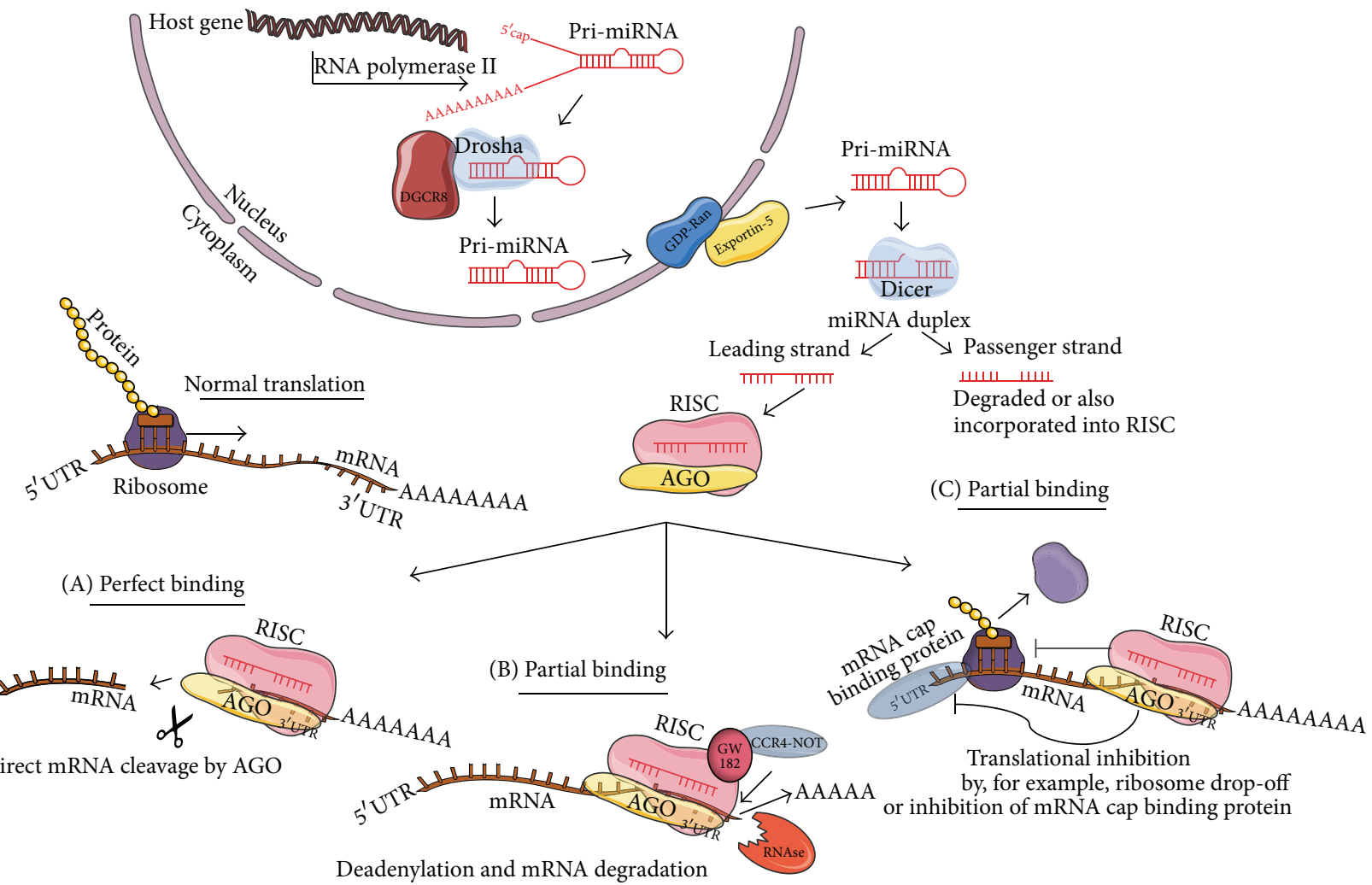

FIGURE 1: miRNA biogenesis. miRNAs are transcribed as mRNA transcripts from the genome by polymerase II as pre-miRs. Endoribonuclease drosha and DGCR8 excise pre-miRs from the primary transcripts. Pri-miRs are exported from the nucleus by exportin-5. An endoribonuclease dicer processes the pre-miRNA and removes the hair loop sequence, creating a double stranded miRNA duplex. One or both strands can be incorporated in RNA-induced silencing complex RISC, which allows the miRNA to suppress translation of their target mRNA or cleave the mRNA and lead to the degradation of it. miRNA-induced RISC can act on their targets by three ways. When there is perfect pairing between the miRNA sequence and its target site, the mRNA is cleaved (A). If the pairing is partial, deadenylation of the mRNA via recruitment of the CCR4-NOT complex takes place and the poly-A tail is lost, leaving the mRNA vulnerable to RNAse activity and mRNA degradation (B). As a second manner of action when pairing is not perfect, the miRNA-induced RISC can also induce repression of translation by blocking initiation or further steps of translation, by mechanisms such as, for example, the promotion of ribosome drop-off from the mRNA transcript or destabilization of the mRNA cap binding protein $(C)$.

(miRBase, version 21, September 2015) [28]. The sequence of miR-378a mature strands is highly conserved between species, with the miR-378a-5p strand being identical in both human and mice and the miR-378a-3p strand only differing in one nucleotide (Table 2) [6, 27].

PGC-1 $\beta$ may regulate several facets of energy metabolism such as mitochondrial biogenesis, thermogenesis, and glucose and fatty acid metabolism [6]. Both strands of miR378 a are coexpressed with PGC-1 $\beta$ as shown, for example, in the liver and during adipocyte differentiation $[6$, 29]. The coexpression of miR-378a with its host gene implies they may share the same transcriptional activators, and miR-378a might be involved in similar processes as PGC-1 $\beta$. Accordingly, high levels of (porcine) miR-378-1 (Table 2) expression are found in developing muscle, postnatal muscle, and myocardium and in brown adipose tissue $[29,30]$.

To date, only a limited number of miR-378a targets, which can be predicted based on in silico analysis, have been experimentally validated. The latter, however, imply a role of miR-378a in mitochondrial energy homeostasis, glycolysis,
TABLE 2: miR-378a is highly conserved between species. Source: miRBase, version 21, September 2015 [28]. The seed sequence (defined as nucleotides 2-8 from the miRNA $5^{\prime}$-end of the mature miRNA) is in bold font.

\begin{tabular}{lcc}
\hline Species & Name & Sequence \\
\hline Human & hsa-miR-378a-5p & $5^{\prime}$-cuccugacuccagguccugugu-3' \\
& hsa-miR-378a-3p & $5^{\prime}$-acuggacuuggagucagaaggc-3' \\
\hline Mouse & $\begin{array}{c}\text { mmu-miR-378a-5p } \\
\text { mmu-miR-378a-3p }\end{array}$ & $5^{\prime}$-acuccugacuccagguccugugu-3' \\
\hline Rat & rno-miR-378a-5p & $5^{\prime}$-cuccugacuccagguccugugu-3' \\
& rno-miR-378a-3p & $5^{\prime}$-acuggacuuggagucagaagg•-3' \\
\hline Pig & ssc-miR-378-1 & $5^{\prime}$-acuggacuuggagucagaaggc-3' \\
\hline Cow & bta-miR-378-1 & $5^{\prime}$-acuggacuuggagucagaaggc-3' \\
\hline $\begin{array}{l}\text { Thirteen- } \\
\text { lined ground } \\
\text { squirrel }\end{array}$ & itr-miR-378a & $5^{\prime}$-acuggacuuggagucagaaggc-3' \\
\hline
\end{tabular}

and skeletal muscle development and in tumor angiogenesis and other processes (Table 3 ). 


\section{4. miR-378a in Metabolism}

A major source of energy production comprises oxidation of glucose in glycolysis followed by oxidation of pyruvate in well-oxygenated cells (or followed by lactic acid fermentation in cancer, the Warburg effect) and from $\beta$-oxidation of lipids, which yields even more ATP per gram then carbohydrates metabolism. A complicated net of metabolic pathways requires advanced regulation by signaling molecules and hormones.

A location of miR-378a in the gene encoding PGC-1 $\beta$ [5] implies an involvement of miR-378a in metabolic pathways. Unlike its homologue, PGC- $1 \alpha$, the expression of PGC- $1 \beta$ is not elevated in response to cold exposure [31] but occurs in response to hypoxia, exercise, caloric restriction, or aging (reviewed in [32]). PGC-1 $\beta$ is preferentially expressed in tissues with relatively high mitochondrial content, such as heart, skeletal muscle, and brown adipose tissue [6]. In 2002, PGC- $1 \beta$ was first cloned and shown to be upregulated in the liver during fasting [31]. PGC- $1 \beta$ strongly activates hepatic nuclear factor 4 (HNF4) and PPAR $\alpha$, both of these nuclear receptors being important for the adaptation of hepatocytes to the effects of fasting. These findings could hint to a possible role of PGC- $1 \beta$ in the regulation of gluconeogenesis and fatty acid oxidation in the liver [31]. PGC-1 $\beta$ is also involved in the regulation of energy expenditure or in the pathway of estrogen receptor-related receptors (ERRs) [3337]. Since miRNAs originating in the introns of host genes may modulate the protein encoded by their parental genes and may be involved in the same mechanisms [38-40], miR$378 \mathrm{a}$ is proposed to be involved in the metabolic pathways affected by PGC-1 $\beta[6]$.

It was reported that mice lacking the first intron of the ppargclb gene (and thus miR-378a) have a significantly higher oxygen capacity and mitochondrial function [6]. Such mice also exhibit a resistance to high fat induced obesity. They identified a mediator complex subunit 13 (MED13), involved in nuclear receptor signaling, and carnitine acetyltransferase (CRAT), a mitochondrial enzyme involved in fatty acid metabolism, as targets of miR-378a-5p and miR$378 \mathrm{a}-3 \mathrm{p}$, respectively [6]. It implies that miR-378a plays a regulatory role in lipid metabolism. miR-378a-5p regulated also cytochrome P450 2E1 (CYP2E1) being involved in the metabolism of, for example, drugs and toxins [41].

In addition, it has been discovered that transcription factor nuclear respiratory factor-1 (NRF-1), a critical regulator of the expression of some important metabolic genes in mitochondria regulating cellular growth, is inhibited by miR$378 \mathrm{a}-3 \mathrm{p}$ [42]. Thus, miR-378a can be considered as a regulator of mitochondrial function in cells overexpressing miR-378a.

Moreover, miR-378a-5p inhibits the mRNAs of ERR $\gamma$ and GA-binding protein- $\alpha$ in breast cancer, which both interact with PGC- $1 \beta$ and together control oxidative metabolism [5]. This leads to a reduction of tricarboxylic acid gene expression and oxygen consumption and an increase in lactate production, which shifts cells from an oxidative towards a glycolytic pathway. In this way, miR-378a-5p is believed to be a switch regulating the Warburg effect in breast cancer [5]. Moreover, in situ hybridization experiments in this study showed that
miR-378a-5p expression correlates with progression of breast cancer [5]. The proposed regulating role of miR-378a-5p on the Warburg effect is in parallel with the effects of PGC- $1 \beta$, which mediates gluconeogenesis and fatty acid metabolism after periods of fasting or intense exercise [31]. Coactivation by PGC- $1 \beta$ of ERR $\alpha$ and PPAR $\alpha$ makes muscle fibers in PGC$1 \beta$ transgenic mice more rich in mitochondria and highly oxidative [43]. Accordingly, such animals were able to run for longer times and at higher workloads [43].

Increased glycolytic rates and increased cell proliferation can be related to lactate production by lactate dehydrogenase (LDH). LDHA was found to be a direct target of miR-378a in the study of Mallat et al. [44]. In this way, hsa-miR-378a$3 p$ represses cell growth and increases cell death by targeting LDHA. Of note, hsa-miR-378a-3p and hsa-miR-378a-5p had opposite effects on LDHA expression. LDHA was significantly downregulated by miR-378a-3p overexpression and upregulated by miR-378a-5p overexpression [44].

In addition, miR-378a is also considered as an important factor in adipogenesis and lipid storage. There is a complex family of factors regulating those processes such as insulin [45], insulin-like growth factors (IGFs), glucagon, and thyroid hormones T3 and T4 (reviewed in [46-49]). As mentioned before, it was demonstrated that miR-378aknockout mice do not get fat after 8 weeks of high fat diet [6]. Such animals show an enhanced mitochondrial fatty acid metabolism and have elevated oxidative capacity of tissues targeted by insulin (e.g., liver, muscles, and adipose tissues) [6]. In accordance with that, it was shown that mature strands of bta-miR-378-1 (Table 1) are expressed at higher level in cows with high (versus low) amount of back fat [50]. Similarly, an inhibition of both mmu-miR-378a-3p and its host gene, PGC- $1 \beta$, by the flavonoid fisetin lowered the accumulation of fat in the liver [42]. Interestingly, mmumiR-378a-5p was downregulated in mice that were fed a high fat diet for five months [51]. In addition, miR-378a is highly induced during adipogenesis [29]. Overexpression of miR-378a-3p/-5p during adipogenesis increased the transcriptional activity of CCAAT/enhancer-binding proteins (cEBP) alpha and beta, which can stimulate the expression of leptin, a hormone produced mainly by adipocytes which controls the homeostasis of body weight [29] (reviewed in $[52,53])$. On the other hand, TNF- $\alpha$, IL-6, and leptin are reported to increase the expression of miR-378a-3p in mature human adipocytes in vitro [54]. These cytokines are mainly secreted in the adipose tissue and are suggested to be involved in development of insulin resistance $[55,56]$. In addition, miR-378a-3p was shown to target insulin growth factor 1 receptor (IGF1R) and reduce the Akt signaling cascade in cardiomyocytes during cardiac development [57]. Moreover, in tissues where IGF1 levels were high (e.g., fibroblasts and fetal hearts), miR-378-3p levels were very low, showing an inverse relation and suggesting a negative feedback loop between miR-378a-3p, IGF1R, and IGF1 [57].

As already mentioned, PGC- $1 \beta$ is a coactivator of PPAR $\gamma$ [5]. The latter functions as a master regulator of adipogenesis and is involved in the formation of peroxisomes and the catabolism of very long chain fatty acids [58, 59]. PPAR $\gamma$ facilitates also the storage of fat in part by inhibiting leptin 


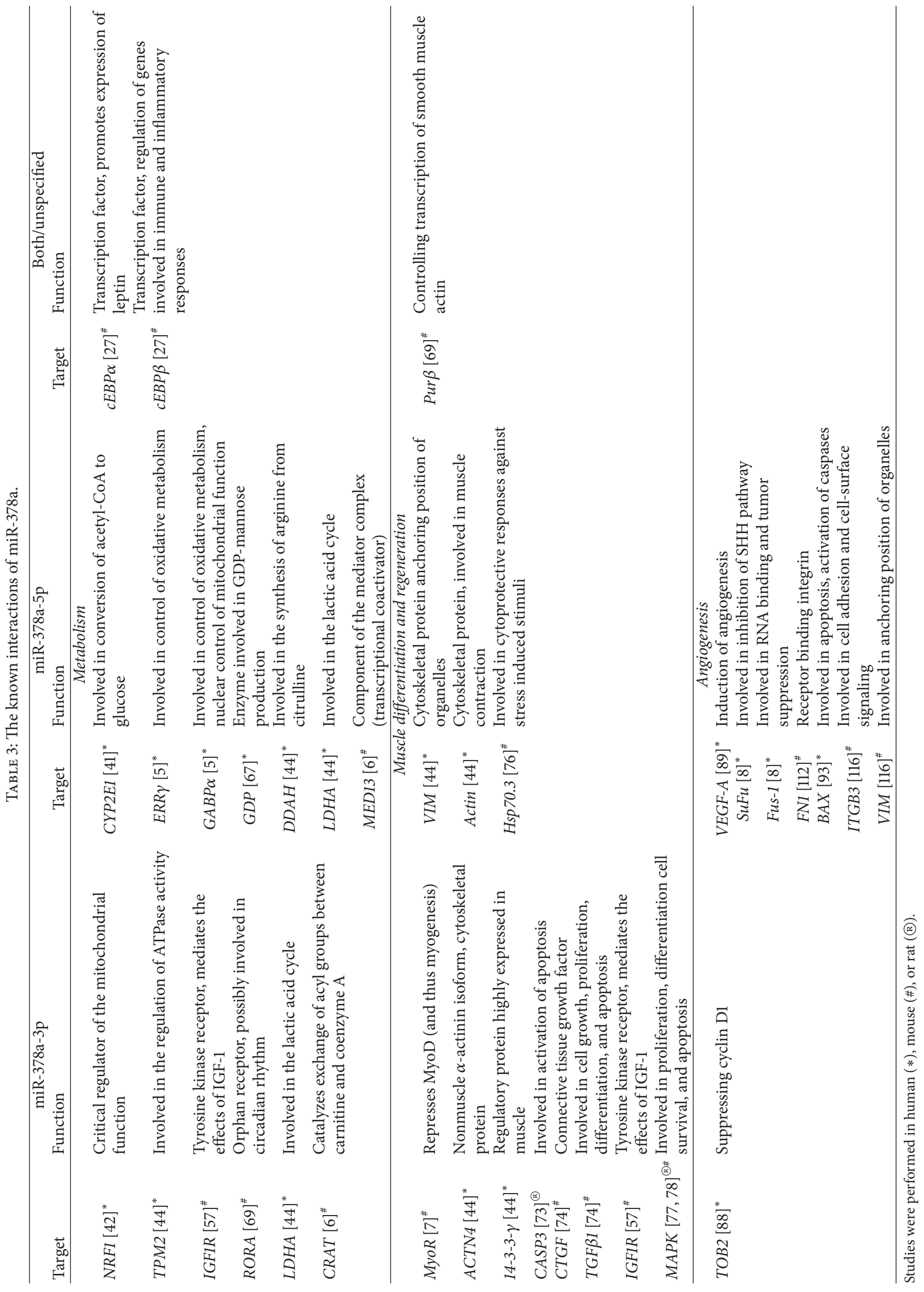


[60]. Accordingly, the amount of adipose tissue does not increase in mice lacking PPAR $\gamma$ when they are fed a high fat diet [61]. It was also reported that in cultured adipocytes mmu-miR-378a and PGC- $1 \beta$ expression is PPAR $\gamma$, or rosiglitazone (a PPAR $\gamma$ ligand), dependent, finding two peroxisome proliferator response elements in the miR-378a loci [62]. On the other hand, overexpression of miR-378a elevated the expression of PPAR $\gamma$ isoform 2 [29], suggesting positive feedback loop and confirming the involvement of miR-378a in the storage of fat.

There are several activators known to induce expression of PPAR $\gamma$ such as the members of the E2F transcription factor family and prostaglandin J-2 (PGJ-2) [63-65]. The latter may act through RAR-related orphan receptor alpha (RORA), which is frequently found in myocardium [66]. In addition to PPAR $\gamma$, RORA regulates also MyoD, a major transcription factor involved in skeletal muscle differentiation [67, 68]. Interestingly, RORA is a possible (but not yet validated) target for miR-378a-3p [69].

A proteomics-based study revealed several other proteins that are potentially targeted by rat miR-378a-3p or miR378a-5p. miR-378a-3p was shown to regulate mannose1-phosphate guanylyltransferase (GDP), dimethylarginine dimethylaminohydrolase 1 (DDAH1), and lactate dehydrogenase A (LDHA); all those proteins are participating in metabolic processes [44]. On the other hand, tropomyosin beta chain, which is involved in the regulation of ATPase activity, was found to be a target of miR-378a-5p [44].

\section{5. miR-378a in Muscle Development, Differentiation, and Regeneration}

High levels of murine and rat miR-378a-3p, miR-378a-5p, and porcine miR-378-1 are reported in both developing and adult skeletal muscles $[7,30,44]$. miR-378a expression is enhanced during skeletal muscle differentiation [30].

MyoD and MyoG play a role in the processes of myogenesis and muscle regeneration, in which dormant satellite cells are activated upon muscle damage and start proliferating and differentiating into muscle fibers (reviewed in [70, 71]). It has been shown that miR-378a-3p targets the myogenic repressor MyoR during myoblast differentiation, which directly inhibits MyoD [7]. On the other hand, MyoD is upregulated in response to $\mathrm{miR}-378 \mathrm{a}-3 \mathrm{p}$ overexpression and, conversely, the level of miR-378a-3p may be enhanced by MyoD [7]. Thus, there is evidence for a feedback loop in which miR$378 \mathrm{a}-3 \mathrm{p}$ regulates muscle differentiation via inhibiting MyoR, leading to an increase of $\mathrm{MyoD}$, which in turn enhances miR$378 a-3 p$ [7].

It has been suggested by Davidsen et al. that miR378a may also control the development of skeletal muscle mass after training [72]. In this study, miR-378a (strand not specified) was significantly downregulated in men who obtained low training-induced muscle mass gain compared to men who obtained high training-induced muscle mass gain [72].

A growing body of data shows a role of miR-378a-3p in the myocardium. miR-378a-3p is expressed mostly by cardiomyocytes, but not by nonmuscle cells, whereas the level of miR-378a-5p was reported to be very low in the heart [57]. Fang et al. showed that miR-378-3p is significantly downregulated both in vitro in cardiomyocytes cell cultures exposed to hypoxia and in vivo during myocardial injury in rats [73]. Overexpression of miR-378a-3p enhanced cell viability and inhibited apoptosis via caspase-3 inhibition [73]. In contrast to this finding, another study found that miR378a-3p downregulation enhanced the survival of cardiac stem cells via focal adhesion kinase activation and releasing connective tissue growth factor (CTGF), the latter being a target of miR-378a-3p [74]. miR-378a inhibition enhanced cardiomyocytes survival after $\mathrm{H}_{2} \mathrm{O}_{2}$ treatment [57]. Overexpression of miR-378a-3p in the study of Knezevic et al. increased apoptosis of cardiomyocytes via the direct targeting of IGF1R leading to a decrease of Akt signaling [57]. This is in opposition to the previously mentioned study of Fang et al. which showed apoptosis was decreased during miR378a-3p overexpression due to targeting of caspase-3 [73]. The converse findings of the studies could be explained by different models used by Knezevic et al. and Fang et al. Because of those discrepancies, the role of miR-378a in apoptosis of cardiomyocytes requires further investigation. The finding that miR-378a-3p affects both IGF1R and the Akt pathway was confirmed [75] in a study which found that overexpression of miR-378a-3p in rhabdomyosarcoma suppressed IGF1R expression and affected phosphorylation of the Akt protein [75]. miR-378a-5p was shown to target heat shock protein 70.3 (Hsp70.3) in mouse hearts in normoxic conditions, but in hypoxic conditions a transcript variant of Hsp70.3 without miR-378a-5p target site in its $3^{\prime}$-UTR is not repressed and can exert its cytoprotective properties [76].

Potential involvement of miR-378a in cardiac remodeling was also proposed. miR-378a-3p prevented cardiac hypertrophy by targeting either Ras signaling or the mitogen-activated protein kinase (MAPK) pathway $[77,78]$.

More studies on the effect of miR-378a expression in muscle disorders would also be desirable. In both Golden Retriever muscular dystrophy dogs and Duchenne muscular dystrophy patients, miR-378a expression was dysregulated, suggesting some relation between miR-378a expression and muscle dystrophy [79].

All in all, these findings suggest miR-378a-3p can be considered as an important player in cardiac development, remodeling, and hypertrophy.

\section{6. miR-378a in Angiogenesis}

Angiogenesis comprises development of new blood vessels from existing ones, regulated by cytokines and growth factors such as, for example, vascular endothelial growth factor (VEGF), platelet-derived growth factor (PDGF), and angiopoietin-1 (Ang-1). Their expression can be posttranscriptionally controlled by microRNAs such as miR-126, miR-296, miR-210, miR-21, and the miR-17 92 cluster [80] (reviewed in [81]).

Skeletal muscles and heart muscle are tissues which, due to their oxygen and energy consumption, need to be sufficiently vascularized. One of the major regulators of angiogenesis is the hypoxia-inducible factor-1 (HIF-1), which 
controls over 100 genes [82] involved mainly in the glycolytic pathway and blood vessel formation, including VEGF-A or interleukin-8 [83-85]. VEGF is generally induced by hypoxia, while IL- 8 in at least some cancers and endothelial cells can be diminished by HIF-1 via inhibition of c-Myc and Sp-1 transcription factors [86, 87]. c-Myc, known as a regulator of cell cycle progression, apoptosis, and cellular transformation, is also a potent activator of PGC- $1 \beta$ and, in turn, miR-378a$3 p$, upregulating their expression [88].

In addition, miR-378a has been shown to affect VEGFA in two ways. Human hsa-miR-378a-5p (by the study of Hua et al. named as miR-378) can directly affect VEGF-A by competing with hsa-miR-125a for the same seed-region in the VEGF-A $3^{\prime}$ UTR causing upregulation of VEGF-A [89]. miR-378a-5p can also indirectly regulate VEGF-A affecting sonic hedgehog $(\mathrm{SHH})$ signaling via Sufu inhibition, which is an inhibitory component of this signaling pathway [8]. The $\mathrm{SHH}$ pathway in turn can upregulate VEGF-A and also other regulators of blood vessels formation, Ang-1 and Ang-2 expression [90-92]. Increased expression of VEGF-A, as well as PDGF $\beta$ and TGF $\beta 1$, was also seen in mesenchymal stromal cells (MSCs) transfected with rno-miR-378a-5p [93].

In skeletal muscles, VEGF-induced angiogenesis appears not to be regulated by the well-known HIF pathway but by PGC- $1 \alpha$, which coactivates estrogen-related receptor alpha $(\mathrm{ERR}-\alpha)$ on binding sites in the promoter and the first intron of the VEGF gene, inducing its expression [94]. This angiogenic pathway shows new roles for PGC- $1 \alpha$ and ERR$\alpha$, which are important regulators of mitochondrial activity in response to stimuli like exercise. If there might be a role for PGC- $1 \beta$ in this pathway, it is yet to be examined. It is noteworthy, however, that miR-378a-5p is known to affect the estrogen receptors by inhibiting $\operatorname{ERR} \gamma$, another estrogenrelated receptor [5].

A role for miR-378a in cell cycle regulation and stimulation of cell growth is also proposed. In human mammary epithelial and breast cancer cell lines, miR-378a-3p can target the antiproliferative protein TOB2, which is a suppressor of cyclin D1, which in turn is required for cell cycle G1phase to S-phase progression [88]. Enhancing endothelial cell proliferation via cell cycle regulation contributes to the angiogenic process. Whether miR-378 affects endothelial cell proliferation by regulation of cell cycle remains to be established.

The role of miR-378a in the formation of blood vessels nourishing tumor and enabling tumor growth was revealed. miR-378a was found to be differentially regulated in different types of cancers [95] being downregulated in gastric cancer [96, 97], oral [98], and colon carcinoma [99], while being upregulated in renal [100] and lung cancer [9, 101]. Since it is also changed in serum or plasma of patients with prostate cancer [102], renal cancer [100, 103], and gastric cancer [104] and frequently found to be overexpressed in cryopreserved bone marrow mononuclear cells from acute myeloid leukemia patients [105], miR-378a might be considered as a biomarker.

The role of miR-378a in tumorigenesis, tumor growth, and tumor vascularization was revealed for the first time by Lee and coworkers in glioblastoma [8]. They showed that miR-378a-5p enhances cell survival, reduces caspase3 activity, and promotes tumor growth and angiogenesis, through repression of two tumor suppressors, Sufu and Fus-1 [8]. Strikingly, nude mice injected with miR-378a-5p transfected cancer cells formed tumors of bigger volume and with larger blood vessels compared to GFP-transfected cells. On the other hand, high expression of miR-378a-5p in NSCLC correlated with brain metastases due to higher cell migration, invasion, and tumor angiogenesis [9]. Another study confirmed the downregulation of Fus-1 by miR-378a-5p and showed that in the HepG2 liver cancer cells miR-378a-5p overexpression enhanced proliferation, migration, and, when injected in mice, invasion [106]. Also in rhabdomyosarcoma, enhanced expression of miR-378a, VEGF, and MMP9 correlated with increased vascularization and metastasis [107]. Taken together, these studies suggest that miR-378a may serve as a prognostic marker in cancer due to its effects on angiogenesis.

Our recent data confirmed the proangiogenic effect of miR-378a (both strands) in non-small cell lung carcinoma (NSCLC) and pointed at its correlation with heme-degrading enzyme, heme oxygenase-1 (HO-1). An involvement of $\mathrm{HO}-$ 1 in angiogenesis and VEGF-A as well as IL-8 signaling was shown by us previously [108]; however, its action in tumors seems to be complex [109]. In NCI-H292 cell line overexpressing HO-1, miR-378a (both strands) levels decreased [101]. Conversely, when HO-1 was silenced using siRNA, miR-378a expression was enhanced. Also overexpression of the miR-378a precursor sequence diminished HO-1 expression. Conditioned medium from NCI-H292 cells overexpressing miR-378a enhanced angiogenic potential of HMEC-1 endothelial cell line. Tumors formed by such cells in subcutaneous xenografts showed enhanced growth, vascularization, oxygenation, and distal metastasis in vivo [101]. These interactions between miR-378a and HO-1 were confirmed in our studies on the role of the Nrf-2 transcription factor/HO-1 axis in NSCLC cell lines [110, 111].

On the other hand, enhanced expression of mmu-miR378a-5p in 4T1 murine breast cancer cells decreased the proliferation, migration, and invasiveness of these cancer cells in vitro and in vivo by targeting fibronectin, resulting in inhibition of tumor growth [112].

Recent study showed that miR-378a may act as a biomarker for response to antiangiogenic treatment in ovarian cancer [113]. Low expression of miR-378a was associated with longer progressive-free survival in patients with recurrent ovarian cancer treated with the antiangiogenic drug bevacizumab [113]. Overexpression of the miR-378a precursor in ovarian cancer cells altered expression of genes associated with angiogenesis (ALCAM, EHD1, ELK3, and TLN1), apoptosis (RPN2, HIPK3), and cell cycle regulation (SWAP-70, LSM14A, and RDX) [113]. High miR-378a (strand not specified) expression in renal carcinoma correlated with higher levels of endothelial surface marker CD34 in these tumors [114].

Notably, a recent study suggested clinical relevance for miR-378a in metastatic colorectal cancer, in which enhanced miR-378a expression significantly improved the sensitivity to cetuximab treatment in these patients [115]. 
Interestingly, recent data indicate a role of miR-378a in stem cells. miR-378a-5p transfection of MSCs has been shown to enhance their survival and angiogenic potential under hypoxic conditions in vitro [93]. In coculture with human umbilical vein endothelial cells (HUVECs), miR378a-5p-transfected MSCs formed a larger number of vascular branches on Matrigel. In the MSCs transfected with miR$378 \mathrm{a}-5 \mathrm{p}$, the expression of $\mathrm{Bcl}-2$-associated $\mathrm{X}$ protein (BAX), which is an important proapoptotic regulator, was decreased, leading to a better survival [93].

It still has to be determined if the proangiogenic effect of miR-378a in vivo is confined to tumor angiogenesis, or if this effect is also present in physiological angiogenesis and regenerative neovascularization. Interestingly, new findings in wound healing studies found a rather opposite conclusion. Recently, it was reported that anti-miR-378a-5p enhances wound healing process by upregulating integrin beta- 3 and vimentin [116].

The role of the host gene of miR-378a on angiogenesis has also been studied. PGC- $1 \beta$ was reported to have opposite effects in ischemia-induced angiogenesis. It was reported that PGC- $1 \beta$ induces angiogenesis in skeletal muscle, enhancing the expression of VEGF both in vitro and in vivo after (transgenic) overexpression [117]. Accordingly, it was also found that VEGFA is upregulated in $\mathrm{C} 2 \mathrm{C} 12$ myoblast cell line with PGC-1 $\beta$ overexpression. However, after a PCRbased gene array of 84 known angiogenic factors and further RT-PCR of individual genes, they concluded that PGC- $1 \beta$ triggered an antiangiogenic program [118]. After inducing hind limb ischemia in PGC- $1 \beta$ overexpressing mice, an impaired reperfusion was noticed when compared to wild type littermates [118].

\section{7. miR-378a in Inflammation}

The role of inflammation in angiogenesis is studied the most in the context of cancer (e.g., reviewed in $[119,120]$ ) but is certainly not limited to this pathology. Both lymphoid (reviewed in [121, 122]) and myeloid (reviewed in [123]) derived inflammatory cells affect angiogenesis in a stimulating or inhibitory manner. The role of miR-378a in inflammatory cells was reported and its anti-inflammatory effect could be suggested.

NK cells exert potent cytotoxic effects when activated by type I IFN from the host once infected [124]. miR-378a was found to be downregulated in activated NK cells and further proved to target granzyme B. Thus, IFN- $\alpha$ activation decreases miR-378a expression and in turn augments NK cell cytotoxicity [124]. Accordingly, suppression of miR-378 targeting granzyme B in NK cells resulted in inhibition of Dengue virus replication in vivo [125].

Macrophages are known to play either inhibitory or stimulatory roles in angiogenesis (reviewed by [126]). miRNAs have been proposed to regulate activation and polarization of macrophages (reviewed by $[127,128]$ ). In a study of Rückerl et al. miR-378a-3p was identified as a part of the IL-4-driven activation program of anti-inflammatory macrophages (M2) [129]. miR-378a-3p was highly upregulated after stimulation with IL-4 of peritoneal exudate cells of mice injected with the parasite Brugia malayi compared to controls and infected IL-4-knockout mice. The study identified several targets for miR-378a-3p within the PI3 K/Akt signaling pathway, which are important for proliferation but only partially responsible for M2 phenotype [129]. Another study found miR-378a (strand not specified) expression upregulated after stimulation with cytokines like, for example, TNF- $\alpha$ and IL- 6 [130].

In line with its potential role in macrophages, miR-378a has been suggested as being of importance in the osteoclastogenesis [131]. Mmu-miR-378a (strand not specified) has been found to be upregulated during osteoclastogenesis in vitro [131]. Furthermore, serum levels of miR-378a-3p have been shown to correlate with bone metastasis burden in mice injected with mouse mammary tumor cell lines 4T1 and 4T1.2 [132].

\section{Conclusions}

A growing body of evidence suggests a role for miR-378a as a mediator controlling reciprocally dependent processes in metabolism, muscle differentiation/regeneration, and angiogenesis.

As miR-378a was found to be differentially regulated in different types of cancers and its level is changed in serum of prostate, renal, and gastric cancer patients, it can be considered as a biomarker for those diseases. The correlation between miR-378a expression and disease progression in lung cancer, liver cancer, and rhabdomyosarcoma suggests a further role of this microRNA as a prognostic marker.

Currently, miR-378a is not utilized as a therapeutic molecule. However, if more research will be done to the mechanisms of action, possibilities for therapeutic use of miR-378a could be sought in the field of metabolic disorders, obesity, or tumors. More studies on the effect of miR-378a expression in muscle disorders would also be desirable.

The proangiogenic effect of miR-378a was observed in tumors; however, no studies have been performed on the angiogenic effects of miR-378a in physiological settings or diseases where angiogenesis plays important roles, such as diabetes and cardiovascular diseases. More study has to be done to assess the mechanisms of miR-378a function in blood vessel formation. Of note, in contrast with proangiogenic role of miR-378a, inhibition of miR-378a-5p enhanced wound healing process. This might suggest a role for miR-378a-5p in diseases such as diabetes or in decubitus ulcers, in which wound healing is impaired.

Of note is the confusion that has arises because of a disarray in nomenclature with studies describing the same molecule, miR-378a, as miR-422b, miR-378, or miR-378* . In addition, it is not always clear which of the two mature strands of miR-378a is studied. This could lead to misunderstandings and errors in interpreting the data published so far.

\section{Disclosure}

The graphical art (Figure 1) was performed with the use of Servier Medical Art. 


\section{Conflict of Interests}

The authors declare that there is no conflict of interests regarding the publication of this paper.

\section{Acknowledgments}

This work was supported by the MAESTRO (2012/06/A/NZ1/ 0004) and OPUS (2012/07/B/NZ1/0288) grants of the Polish National Science Centre and the Iuventus Plus (0244/IP1/ 2013/72) from the Ministry of Science and Higher Education. Faculty of Biochemistry, Biophysics and Biotechnology is a partner of the Leading National Research Center (KNOW) supported by the Ministry of Science and Higher Education.

\section{References}

[1] Z. Wang, Z. Ying, A. Bosy-Westphal et al., "Specific metabolic rates of major organs and tissues across adulthood: evaluation by mechanistic model of resting energy expenditure," The American Journal of Clinical Nutrition, vol. 92, no. 6, pp. 13691377, 2010.

[2] K. L. Blaxter, Energy Metabolism in Animals and Man, Cambridge University Press, Cambridge, UK, 1989.

[3] D. Gallagher, D. Belmonte, P. Deurenberg et al., "Organ-tissue mass measurement allows modeling of REE and metabolically active tissue mass," American Journal of Physiology, vol. 275, no. 2, part 1, pp. E249-E258, 1998.

[4] M. N. Poy, M. Spranger, and M. Stoffel, "microRNAs and the regulation of glucose and lipid metabolism," Diabetes, Obesity and Metabolism, vol. 9, supplement 2, pp. 67-73, 2007.

[5] L. J. Eichner, M. C. Perry, C. R. Dufour et al., "miR-378 $\left({ }^{*}\right)$ mediates metabolic shift in breast cancer cells via the PGC1 $\beta$ /ERR $\gamma$ transcriptional pathway," Cell Metabolism, vol. 12, no. 4, pp. 352-361, 2010.

[6] M. Carrer, N. Liu, C. E. Grueter et al., "Control of mitochondrial metabolism and systemic energy homeostasis by microRNAs 378 and 378*", Proceedings of the National Academy of Sciences of the United States of America, vol. 109, no. 38, pp. 15330-15335, 2012.

[7] J. Gagan, B. K. Dey, R. Layer, Z. Yan, and A. Dutta, "MicroRNA378 targets the myogenic repressor MyoR during myoblast differentiation," The Journal of Biological Chemistry, vol. 286, no. 22, pp. 19431-19438, 2011.

[8] D. Y. Lee, Z. Deng, C.-H. Wang, and B. B. Yang, "MicroRNA378 promotes cell survival, tumor growth, and angiogenesis by targeting $\mathrm{SuFu}$ and Fus-1 expression," Proceedings of the National Academy of Sciences of the United States of America, vol. 104, no. 51, pp. 20350-20355, 2007.

[9] L.-T. Chen, S.-D. Xu, H. Xu, J.-F. Zhang, J.-F. Ning, and S.-F. Wang, "MicroRNA-378 is associated with non-small cell lung cancer brain metastasis by promoting cell migration, invasion and tumor angiogenesis," Medical Oncology, vol. 29, no. 3, pp. 1673-1680, 2012.

[10] I. Lee, S. S. Ajay, I. Y. Jong et al., "New class of microRNA targets containing simultaneous $5^{\prime}$-UTR and $3^{\prime}$-UTR interaction sites," Genome Research, vol. 19, no. 7, pp. 1175-1183, 2009.

[11] R. C. Lee, R. L. Feinbaum, and V. Ambros, "The C. elegans heterochronic gene lin-4 encodes small RNAs with antisense complementarity to lin-14," Cell, vol. 75, no. 5, pp. 843-854, 1993.
[12] B. P. Lewis, C. B. Burge, and D. P. Bartel, "Conserved seed pairing, often flanked by adenosines, indicates that thousands of human genes are microRNA targets," Cell, vol. 120, no. 1, pp. 15-20, 2005.

[13] I. Bentwich, "Prediction and validation of microRNAs and their targets," FEBS Letters, vol. 579, no. 26, pp. 5904-5910, 2005.

[14] R. C. Friedman, K. K.-H. Farh, C. B. Burge, and D. P. Bartel, "Most mammalian mRNAs are conserved targets of microRNAs," Genome Research, vol. 19, no. 1, pp. 92-105, 2009.

[15] S.-Y. Ying and S.-L. Lin, "Intronic microRNAs," Biochemical and Biophysical Research Communications, vol. 326, no. 3, pp. 515520, 2005.

[16] S. Baskerville and D. P. Bartel, "Microarray profiling of microRNAs reveals frequent coexpression with neighboring miRNAs and host genes," RNA, vol. 11, no. 3, pp. 241-247, 2005.

[17] Y. Lee, M. Kim, J. Han et al., "MicroRNA genes are transcribed by RNA polymerase II," The EMBO Journal, vol. 23, no. 20, pp. 4051-4060, 2004.

[18] Y. Altuvia, P. Landgraf, G. Lithwick et al., "Clustering and conservation patterns of human microRNAs," Nucleic Acids Research, vol. 33, no. 8, pp. 2697-2706, 2005.

[19] E. P. Murchison and G. J. Hannon, "miRNAs on the move: miRNA biogenesis and the RNAi machinery," Current Opinion in Cell Biology, vol. 16, no. 3, pp. 223-229, 2004.

[20] A. E. Pasquinelli, "MicroRNAs and their targets: recognition, regulation and an emerging reciprocal relationship," Nature Reviews Genetics, vol. 13, no. 4, pp. 271-282, 2012.

[21] D. P. Bartel, "MicroRNAs: genomics, biogenesis, mechanism, and function," Cell, vol. 116, no. 2, pp. 281-297, 2004.

[22] K. Kasashima, Y. Nakamura, and T. Kozu, "Altered expression profiles of microRNAs during TPA-induced differentiation of HL-60 cells," Biochemical and Biophysical Research Communications, vol. 322, no. 2, pp. 403-410, 2004.

[23] M. S. Stark, S. Tyagi, D. J. Nancarrow et al., "Characterization of the melanoma miRNAome by deep sequencing," PLoS ONE, vol. 5, no. 3, Article ID e9685, 2010.

[24] D. D. Jima, J. Zhang, C. Jacobs et al., "Deep sequencing of the small RNA transcriptome of normal and malignant human B cells identifies hundreds of novel microRNAs," Blood, vol. 116, no. 23, pp. el18-e127, 2010.

[25] E. Berezikov, G. Van Tetering, M. Verheul et al., "Many novel mammalian microRNA candidates identified by extensive cloning and RAKE analysis," Genome Research, vol. 16, no. 10, pp. 1289-1298, 2006.

[26] L. A. Goff, J. Davila, M. R. Swerdel et al., "Ago2 immunoprecipitation identifies predicted MicroRNAs in human embryonic stem cells and neural precursors," PLoS ONE, vol. 4, no. 9, Article ID e7192, 2009.

[27] M. Dannemann, K. Prüfer, E. Lizano, B. Nickel, H. A. Burbano, and J. Kelso, "Transcription factors are targeted by differentially expressed miRNAs in primates," Genome Biology and Evolution, vol. 4, no. 4, pp. 552-564, 2012.

[28] A. Kozomara and S. Griffiths-Jones, "MiRBase: integrating microRNA annotation and deep-sequencing data," Nucleic Acids Research, vol. 39, no. 1, pp. D152-D157, 2011.

[29] I. Gerin, G. T. Bommer, C. S. McCoin, K. M. Sousa, V. Krishnan, and O. A. MacDougald, "Roles for miRNA-378/378* in adipocyte gene expression and lipogenesis," American Journal of Physiology-Endocrinology and Metabolism, vol. 299, no. 2, pp. E198-E206, 2010. 
[30] X. Hou, Z. Tang, H. Liu, N. Wang, H. Ju, and K. Li, “Discovery of MicroRNAs associated with myogenesis by deep sequencing of serial developmental skeletal muscles in pigs," PLoS ONE, vol. 7, no. 12, Article ID e52123, 2012.

[31] J. Lin, P. Puigserver, J. Donovan, P. Tarr, and B. M. Spiegelman, "Peroxisome proliferator-activated receptor $\gamma$ coactivator $1 \beta$ (PGC-1 $\beta$ ), a novel PGC-1-related transcription coactivator associated with host cell factor," The Journal of Biological Chemistry, vol. 277, no. 3, pp. 1645-1648, 2002.

[32] I. S. Patten and Z. Arany, "PGC-1 coactivators in the cardiovascular system," Trends in Endocrinology and Metabolism, vol. 23, no. 2, pp. 90-97, 2012.

[33] Y. Kamei, H. Ohizumi, Y. Fujitani et al., "PPAR $\gamma$ coactivator $1 \beta /$ ERR ligand 1 is an ERR protein ligand, whose expression induces a high-energy expenditure and antagonizes obesity," Proceedings of the National Academy of Sciences of the United States of America, vol. 100, no. 21, pp. 12378-12383, 2003.

[34] J. Lin, R. Yang, P. T. Tarr et al., "Hyperlipidemic effects of dietary saturated fats mediated through PGC- $1 \beta$ coactivation of SREBP," Cell, vol. 120, no. 2, pp. 261-273, 2005.

[35] J. Lin, P. T. Tarr, R. Yang et al., "PGC-1 $\beta$ in the regulation of hepatic glucose and energy metabolism," The Journal of Biological Chemistry, vol. 278, no. 33, pp. 30843-30848, 2003.

[36] C. Wolfrum and M. Stoffel, "Coactivation of Foxa2 through Pgc$1 \beta$ promotes liver fatty acid oxidation and triglyceride/VLDL secretion," Cell Metabolism, vol. 3, no. 2, pp. 99-110, 2006.

[37] J. St-Pierre, J. Lin, S. Krauss et al., "Bioenergetic analysis of peroxisome proliferator-activated receptor $\gamma$ coactivators $1 \alpha$ and $1 \beta$ (PGC- $1 \alpha$ and PGC-1 $\beta$ ) in muscle cells," The Journal of Biological Chemistry, vol. 278, no. 29, pp. 26597-26603, 2003.

[38] E. M. Small and E. N. Olson, "Pervasive roles of microRNAs in cardiovascular biology," Nature, vol. 469, no. 7330, pp. 336-342, 2011.

[39] E. van Rooij, D. Quiat, B. A. Johnson et al., "A family of microRNAs encoded by myosin genes governs myosin expression and muscle performance," Developmental Cell, vol. 17, no. 5, pp. 662673, 2009.

[40] S. Wang, A. B. Aurora, B. A. Johnson et al., "The endothelialspecific microRNA miR-126 governs vascular integrity and angiogenesis," Developmental Cell, vol. 15, no. 2, pp. 261-271, 2008.

[41] T. Mohri, M. Nakajima, T. Fukami, M. Takamiya, Y. Aoki, and T. Yokoi, "Human CYP2E1 is regulated by miR-378," Biochemical Pharmacology, vol. 79, no. 7, pp. 1045-1052, 2010.

[42] T.-I. Jeon, J. W. Park, J. Ahn, C. H. Jung, and T. Y. Ha, "Fisetin protects against hepatosteatosis in mice by inhibiting miR-378," Molecular Nutrition \& Food Research, vol. 57, no. 11, pp. 19311937, 2013.

[43] Z. Arany, N. Lebrasseur, C. Morris et al., "The transcriptional coactivator PGC-1beta drives the formation of oxidative type IIX fibers in skeletal muscle," Cell Metabolism, vol. 5, no. 1, pp. 35-46, 2007.

[44] Y. Mallat, E. Tritsch, R. Ladouce et al., "Proteome modulation in H9c2 cardiac cells by microRNAs miR-378 and miR-378," Molecular and Cellular Proteomics, vol. 13, no. 1, pp. 18-29, 2014.

[45] G. Ailhaud, P. Grimaldi, and R. Négrel, "Hormonal regulation of adipose differentiation," Trends in Endocrinology and Metabolism, vol. 5, no. 3, pp. 132-136, 1994.

[46] C. Villar-Palasi, "The hormonal regulation of glycogen metabolism in muscle," Vitamins and Hormones, vol. 26, pp. 65-118, 1968.
[47] R. P. Brun, J. B. Kim, E. Hu, S. Altiok, and B. M. Spiegelman, "Adipocyte differentiation: a transcriptional regulatory cascade," Current Opinion in Cell Biology, vol. 8, no. 6, pp. 826832, 1996.

[48] M. I. Lefterova and M. A. Lazar, "New developments in adipogenesis," Trends in Endocrinology and Metabolism, vol. 20, no. 3, pp. 107-114, 2009.

[49] A. T. Ali, W. E. Hochfeld, R. Myburgh, and M. S. Pepper, "Adipocyte and adipogenesis," European Journal of Cell Biology, vol. 92, no. 6-7, pp. 229-236, 2013.

[50] W. Jin, M. V. Dodson, S. S. Moore, J. A. Basarab, and L. L. Guan, "Characterization of microRNA expression in bovine adipose tissues: a potential regulatory mechanism of subcutaneous adipose tissue development," BMC Molecular Biology, vol. 11, article 29, 2010.

[51] D. V. Chartoumpekis, A. Zaravinos, P. G. Ziros et al., "Differential expression of microRNAs in adipose tissue after long-term high-fat diet-induced obesity in mice," PLoS ONE, vol. 7, no. 4, Article ID e34872, 2012.

[52] S. Collins, C. M. Kuhn, A. E. Petro, A. G. Swick, B. A. Chrunyk, and R. S. Surwit, "Role of leptin in fat regulation," Nature, vol. 380, no. 6576, p. 677, 1996.

[53] P. Trayhurn and D. V. Rayner, "Hormones and the ob gene product (leptin) in the control of energy balance," Biochemical Society Transactions, vol. 24, no. 2, pp. 565-570, 1996.

[54] L.-L. Xu, C.-M. Shi, G.-F. Xu et al., "TNF- $\alpha$, IL-6, and leptin increase the expression of miR-378, an adipogenesis-related microRNA in human adipocytes," Cell Biochemistry and Biophysics, vol. 70, no. 2, pp. 771-776, 2014.

[55] J. M. Stephens, J. Lee, and P. F. Pilch, “Tumor necrosis factor- $\alpha$ induced insulin resistance in 3T3-L1 adipocytes is accompanied by a loss of insulin receptor substrate-1 and GLUT4 expression without a loss of insulin receptor-mediated signal transduction," The Journal of Biological Chemistry, vol. 272, no. 2, pp. 971-976, 1997.

[56] S. Müller, S. Martin, W. Koenig et al., "Impaired glucose tolerance is associated with increased serum concentrations of interleukin 6 and co-regulated acute-phase proteins but not TNF-alpha or its receptors," Diabetologia, vol. 45, no. 6, pp. 805812, 2002.

[57] I. Knezevic, A. Patel, N. R. Sundaresan et al., "A novel cardiomyocyte-enriched MicroRNA, miR-378, targets insulinlike growth factor 1 receptor: implications in postnatal cardiac remodeling and cell survival," The Journal of Biological Chemistry, vol. 287, no. 16, pp. 12913-12926, 2012.

[58] E. D. Rosen and B. M. Spiegelman, "Peroxisome proliferatoractivated receptor $\gamma$ ligands and atherosclerosis: ending the heartache," The Journal of Clinical Investigation, vol. 106, no. 5, pp. 629-631, 2000.

[59] L. Fajas, D. Auboeuf, E. Raspé et al., "The organization, promoter analysis, and expression of the human PPAR $\gamma$ gene," The Journal of Biological Chemistry, vol. 272, no. 30, pp. 1877918789, 1997.

[60] J. R. Zierath and H. Wallberg-Henriksson, Muscle Metabolism, Frontiers in Animal Diabetes Research, Taylor \& Francis, London, UK, 2002.

[61] J. R. Jones, C. Barrick, K.-A. Kim et al., "Deletion of PPAR $\gamma$ in adipose tissues of mice protects against high fat diet-induced obesity and insulin resistance," Proceedings of the National Academy of Sciences of the United States of America, vol. 102, no. 17, pp. 6207-6212, 2005. 
[62] E. John, A. Wienecke-Baldacchino, M. Liivrand, M. Heinäniemi, C. Carlberg, and L. Sinkkonen, "Dataset integration identifies transcriptional regulation of microRNA genes by PPAR $\gamma$ in differentiating mouse 3T3-L1 adipocytes," Nucleic Acids Research, vol. 40, no. 10, pp. 4446-4460, 2012.

[63] L. Fajas, R. L. Landsberg, Y. Huss-Garcia, C. Sardet, J. A. Lees, and J. Auwerx, "E2Fs regulate adipocyte differentiation," Developmental Cell, vol. 3, no. 1, pp. 39-49, 2002.

[64] S. A. Kliewer, J. M. Lenhard, T. M. Willson, I. Patel, D. C. Morris, and J. M. Lehmann, "A prostaglandin $\mathrm{J}_{2}$ metabolite binds peroxisome proliferator-activated receptor $\gamma$ and promotes adipocyte differentiation," Cell, vol. 83, no. 5, pp. 813-819, 1995.

[65] A. Jozkowicz, J. Dulak, E. Piatkowska, W. Placha, and A. Dembinska-Kiec, "Ligands of peroxisome proliferatoractivated receptor-gamma increase the generation of vascular endothelial growth factor in vascular smooth muscle cells and in macrophages," Acta Biochimica Polonica, vol. 47, no. 4, pp. 1147-1157, 2000.

[66] H. Migita and J. Morser, "15-Deoxy- $\Delta 12,14$-postaglandin J2 (15d-PGJ2) signals through retinoic acid receptor-related orphan receptor- $\alpha$ but not peroxisome proliferator-activated receptor- $\gamma$ in human vascular endothelial cells: the effect of 15d-PGJ2 on tumor necrosis factor- $\alpha$-induced gene expression," Arteriosclerosis, Thrombosis, and Vascular Biology, vol. 25, no. 4, pp. 710-716, 2005.

[67] H. Sundvold and S. Lien, "Identification of a novel peroxisome proliferator-activated receptor (PPAR) gamma promoter in man and transactivation by the nuclear receptor RORalphal," Biochemical and Biophysical Research Communications, vol. 287, no. 2, pp. 383-390, 2001.

[68] P. Lau, P. Bailey, D. H. Dowhan, and G. E. O. Muscat, "Exogenous expression of a dominant negative ROR $\alpha 1$ vector in muscle cells impairs differentiation: ROR $\alpha 1$ directly interacts with p300 and MyoD," Nucleic Acids Research, vol. 27, no. 2, pp. 411-420, 1999.

[69] S. J. Matkovich, Y. Hu, and G. W. Dorn II, "Regulation of cardiac microRNAs by cardiac microRNAs," Circulation Research, vol. 113, no. 1, pp. 62-71, 2013.

[70] A. Musarò, "The basis of muscle regeneration," Advances in Biology, vol. 2014, Article ID 612471, 16 pages, 2014.

[71] M. H. Parker, P. Seale, and M. A. Rudnicki, "Looking back to the embryo: defining transcriptional networks in adult myogenesis," Nature Reviews Genetics, vol. 4, no. 7, pp. 497-507, 2003.

[72] P. K. Davidsen, I. J. Gallagher, J. W. Hartman et al., "High responders to resistance exercise training demonstrate differential regulation of skeletal muscle microRNA expression," Journal of Applied Physiology, vol. 110, no. 2, pp. 309-317, 2011.

[73] J. Fang, X.-W. Song, J. Tian et al., "Overexpression of microRNA-378 attenuates ischemia-induced apoptosis by inhibiting caspase- 3 expression in cardiac myocytes," Apoptosis, vol. 17, no. 4, pp. 410-423, 2012.

[74] S. W. Kim, H. W. Kim, W. Huang et al., "Cardiac stem cells with electrical stimulation improve ischaemic heart function through regulation of connective tissue growth factor and miR378," Cardiovascular Research, vol. 100, no. 2, pp. 241-251, 2013.

[75] F. Megiorni, S. Cialfi, H. P. McDowell et al., "Deep sequencing the microRNA profile in rhabdomyosarcoma reveals downregulation of miR-378 family members," BMC Cancer, vol. 14, article 880, 2014.

[76] M. Tranter, R. N. Helsley, W. R. Paulding et al., "Coordinated post-transcriptional regulation of Hsp70.3 gene expression by
microRNA and alternative polyadenylation," The Journal of Biological Chemistry, vol. 286, no. 34, pp. 29828-29837, 2011.

[77] R. S. Nagalingam, N. R. Sundaresan, M. P. Gupta, D. L. Geenen, R. J. Solaro, and M. Gupta, "A cardiac-enriched microRNA, miR-378, blocks cardiac hypertrophy by targeting Ras signaling," The Journal of Biological Chemistry, vol. 288, no. 16, pp. 11216-11232, 2013.

[78] J. Ganesan, D. Ramanujam, Y. Sassi et al., "MiR-378 controls cardiac hypertrophy by combined repression of mitogen-activated protein kinase pathway factors," Circulation, vol. 127, no. 21, pp. 2097-2106, 2013.

[79] L. Jeanson-Leh, J. Lameth, S. Krimi et al., "Serum profiling identifies novel muscle miRNA and cardiomyopathy-related miRNA biomarkers in golden retriever muscular dystrophy dogs and duchenne muscular dystrophy patients," American Journal of Pathology, vol. 184, no. 11, pp. 2885-2898, 2014.

[80] P. Carmeliet and R. K. Jain, "Molecular mechanisms and clinical applications of angiogenesis," Nature, vol. 473, no. 7347, pp. 298307, 2011.

[81] S. Wang and E. N. Olson, "AngiomiRs-Key regulators of angiogenesis," Current Opinion in Genetics and Development, vol. 19, no. 3, pp. 205-211, 2009.

[82] D. J. Manalo, A. Rowan, T. Lavoie et al., "Transcriptional regulation of vascular endothelial cell responses to hypoxia by HIF-1," Blood, vol. 105, no. 2, pp. 659-669, 2005.

[83] J. A. Forsythe, B.-H. Jiang, N. V. Iyer et al., "Activation of vascular endothelial growth factor gene transcription by hypoxiainducible factor 1," Molecular and Cellular Biology, vol. 16, no. 9, pp. 4604-4613, 1996.

[84] A. Loboda, A. Jozkowicz, and J. Dulak, "HIF-1 and HIF-2 transcription factors-similar but not identical," Molecules and Cells, vol. 29, no. 5, pp. 435-442, 2010.

[85] A. Loboda, A. Jozkowicz, and J. Dulak, "HIF-1 versus HIF-2-is one more important than the other?" Vascular Pharmacology, vol. 56, no. 5-6, pp. 245-251, 2012.

[86] U. Florczyk, A. Jazwa, M. Maleszewska et al., "Nrf2 regulates angiogenesis: effect on endothelial cells, bone marrow-derived proangiogenic cells and hind limb ischemia," Antioxidants and Redox Signaling, vol. 20, no. 11, pp. 1693-1708, 2014.

[87] A. Loboda, A. Stachurska, U. Florczyk et al., "HIF-1 induction attenuates Nrf2-dependent IL-8 expression in human endothelial cells," Antioxidants and Redox Signaling, vol. 11, no. 7, pp. 1501-1517, 2009.

[88] M. Feng, Z. Li, M. Aau, C. H. Wong, X. Yang, and Q. Yu, "Myc/miR-378/TOB2/cyclin D1 functional module regulates oncogenic transformation," Oncogene, vol. 30, no. 19, pp. 22422251, 2011.

[89] Z. Hua, Q. Lv, W. Ye et al., "Mirna-directed regulation of VEGF and other angiogenic under hypoxia," PLoS ONE, vol. 1, no. 1, article el16, 2006.

[90] T. Nagase, M. Nagase, K. Yoshimura, T. Fujita, and I. Koshima, "Angiogenesis within the developing mouse neural tube is dependent on sonic hedgehog signaling: possible roles of motor neurons," Genes to Cells, vol. 10, no. 6, pp. 595-604, 2005.

[91] R. Pola, L. E. Ling, M. Silver et al., "The morphogen Sonic hedgehog is an indirect angiogenic agent upregulating two families of angiogenic growth factors," Nature Medicine, vol. 7, no. 6, pp. 706-711, 2001.

[92] K. J. Lavine, A. C. White, C. Park et al., "Fibroblast growth factor signals regulate a wave of Hedgehog activation that is essential for coronary vascular development," Genes and Development, vol. 20, no. 12, pp. 1651-1666, 2006. 
[93] Y. Xing, J. Hou, T. Guo et al., “microRNA-378 promotes mesenchymal stem cell survival and vascularization under hypoxic-ischemic conditions in vitro," Stem Cell Research \& Therapy, vol. 5, no. 6, article 130, 2014.

[94] Z. Arany, S.-Y. Foo, Y. Ma et al., "HIF-independent regulation of VEGF and angiogenesis by the transcriptional coactivator PGC-1 $\alpha$," Nature, vol. 451, no. 7181, pp. 1008-1012, 2008.

[95] J. Jiang, E. J. Lee, Y. Gusev, and T. D. Schmittgen, "Real-time expression profiling of microRNA precursors in human cancer cell lines," Nucleic Acids Research, vol. 33, no. 17, pp. 5394-5403, 2005.

[96] J. Guo, Y. Miao, B. Xiao et al., "Differential expression of microRNA species in human gastric cancer versus nontumorous tissues," Journal of Gastroenterology and Hepatology, vol. 24, no. 4, pp. 652-657, 2009.

[97] Y. Yao, A. L. Suo, Z. F. Li et al., "MicroRNA profiling of human gastric cancer," Molecular Medicine Reports, vol. 2, no. 6, pp. 963-970, 2009.

[98] L. Scapoli, A. Palmieri, L. Lo Muzio et al., "MicroRNA expression profiling of oral carcinoma identifies new markers of tumor progression," International Journal of Immunopathology and Pharmacology, vol. 23, no. 4, pp. 1229-1234, 2010.

[99] Y. X. Wang, X. Y. Zhang, B. F. Zhang, C. Q. Yang, X. M. Chen, and H. J. Gao, "Initial study of microRNA expression profiles of colonic cancer without lymph node metastasis," Journal of Digestive Diseases, vol. 11, no. 1, pp. 50-54, 2010.

[100] M. Redova, A. Poprach, J. Nekvindova et al., "Circulating miR378 and miR-451 in serum are potential biomarkers for renal cell carcinoma," Journal of Translational Medicine, vol. 10, no. 1, article 55, 2012.

[101] K. Skrzypek, M. Tertil, S. Golda et al., "Interplay between heme oxygenase-1 and miR-378 affects non-small cell lung carcinoma growth, vascularization, and metastasis," Antioxidants and Redox Signaling, vol. 19, no. 7, pp. 644-660, 2013.

[102] H. C. N. Nguyen, W. Xie, M. Yang et al., "Expression differences of circulating microRNAs in metastatic castration resistant prostate cancer and low-risk, localized prostate cancer," Prostate, vol. 73, no. 4, pp. 346-354, 2013.

[103] M. Fedorko, M. Stanik, R. Iliev et al., "Combination of MiR-378 and MiR-210 serum levels enables sensitive detection of renal cell carcinoma," International Journal of Molecular Sciences, vol. 16, no. 10, pp. 23382-23389, 2015.

[104] H. Liu, L. Zhu, B. Liu et al., "Genome-wide microRNA profiles identify miR-378 as a serum biomarker for early detection of gastric cancer," Cancer Letters, vol. 316, no. 2, pp. 196-203, 2012.

[105] J. Qian, J. Lin, W. Qian et al., "Overexpression of miR-378 is frequent and may affect treatment outcomes in patients with acute myeloid leukemia," Leukemia Research, vol. 37, no. 7, pp. 765-768, 2013.

[106] J. Ma, J. Lin, J. Qian et al., "MiR-378 promotes the migration of liver cancer cells by down-regulating fus expression," Cellular Physiology and Biochemistry, vol. 34, no. 6, pp. 2266-2274, 2014.

[107] K. Skrzypek, A. Kusienicka, B. Szewczyk et al., "Constitutive activation of MET signaling impairs myogenic differentiation of rhabdomyosarcoma and promotes its development and progression," Oncotarget, vol. 6, no. 31, pp. 31378-31398, 2015.

[108] A. Jazwa, A. Loboda, S. Golda et al., "Effect of heme and heme oxygenase-1 on vascular endothelial growth factor synthesis and angiogenic potency of human keratinocytes," Free Radical Biology and Medicine, vol. 40, no. 7, pp. 1250-1263, 2006.
[109] H. Was, J. Dulak, and A. Jozkowicz, "Heme oxygenase-1 in tumor biology and therapy," Current Drug Targets, vol. 11, no. 12, pp. 1551-1570, 2010.

[110] M. Terti, K. Skrzypek, U. Florczyk et al., "Regulation and novel action of thymidine phosphorylase in non-small cell lung cancer: crosstalk with Nrf2 and HO-1," PLoS ONE, vol. 9, no. 5, Article ID e97070, 2014.

[111] M. Tertil, S. Golda, K. Skrzypek et al., "Nrf2-heme oxygenase1 axis in mucoepidermoid carcinoma of the lung: antitumoral effects associated with down-regulation of matrix metalloproteinases," Free Radical Biology and Medicine, vol. 89, pp. 147-157, 2015.

[112] F. Liu, Q. Lv, W. W. Du et al., "Specificity of miR-378a-5p targeting rodent fibronectin," Biochimica et Biophysica ActaMolecular Cell Research, vol. 1833, no. 12, pp. 3272-3285, 2013.

[113] J. K. Chan, T. K. Kiet, K. Blansit et al., "MiR-378 as a biomarker for response to anti-angiogenic treatment in ovarian cancer," Gynecologic Oncology, vol. 133, no. 3, pp. 568-574, 2014.

[114] H.-C. Li, J.-P. Li, Z.-M. Wang et al., "Identification of angiogenesis-related miRNAs in a population of patients with renal clear cell carcinoma," Oncology Reports, vol. 32, no. 5, pp. 2061-2069, 2014.

[115] W.-H. Weng, W.-H. Leung, Y.-J. Pang, and H.-H. Hsu, "Lauric acid can improve the sensitization of Cetuximab in KRAS/BRAF mutated colorectal cancer cells by retrievable microRNA-378 expression," Oncology Reports, 2015.

[116] H. Li, L. Chang, W. W. Du et al., "Anti-microRNA-378a enhances wound healing process by upregulating integrin beta3 and vimentin," Molecular Therapy, vol. 22, no. 10, pp. 18391850, 2014.

[117] G. C. Rowe, C. Jang, I. S. Patten, and Z. Arany, "PGC-1 $\beta$ regulates angiogenesis in skeletal muscle," American Journal of Physiology: Endocrinology and Metabolism, vol. 301, no. 1, pp. E155-E163, 2011.

[118] V. Yadav, A. Matsakas, S. Lorca, and V. A. Narkar, "PGC1 $\beta$ activates an antiangiogenic program to repress neoangiogenesis in muscle ischemia," Cell Reports, vol. 8, no. 3, pp. 783-797, 2014.

[119] C. Stockmann, D. Schadendorf, R. Klose, and I. Helfrich, "The impact of the immune system on tumor: angiogenesis and vascular remodeling," Frontiers in Oncology, vol. 4, article 69, 2014.

[120] M. Ono, "Molecular links between tumor angiogenesis and inflammation: inflammatory stimuli of macrophages and cancer cells as targets for therapeutic strategy," Cancer Science, vol. 99, no. 8, pp. 1501-1506, 2008.

[121] B. J. Nickoloff, "Characterization of lymphocyte-dependent angiogenesis using a SCID mouse: human skin model of psoriasis," Journal of Investigative Dermatology Symposium Proceedings, vol. 5, no. 1, pp. 67-73, 2000.

[122] A. Naldini, A. Pucci, C. Bernini, and F. Carraro, "Regulation of angiogenesis by Th1- and Th2-type cytokines," Current Pharmaceutical Design, vol. 9, no. 7, pp. 511-519, 2003.

[123] S. E. J. Chambers, C. L. O’Neill, T. M. O’Doherty, R. J. Medina, and A. W. Stitt, "The role of immune-related myeloid cells in angiogenesis," Immunobiology, vol. 218, no. 11, pp. 1370-1375, 2013.

[124] P. Wang, Y. Gu, Q. Zhang et al., "Identification of resting and type I IFN-activated human NK cell miRNomes reveals MicroRNA-378 and MicroRNA-30e as negative regulators of NK cell cytotoxicity," The Journal of Immunology, vol. 189, no. 1, pp. 211-221, 2012. 
[125] S. Liu, L. Chen, Y. Zeng et al., "Suppressed expression of miR378 targeting gzmb in NK cells is required to control dengue virus infection," Cellular \& Molecular Immunology, 2015.

[126] F. O. Martinez, A. Sica, A. Mantovani, and M. Locati, "Macrophage activation and polarization," Frontiers in Bioscience, vol. 13, pp. 453-461, 2008.

[127] G. Liu and E. Abraham, "MicroRNAs in immune response and macrophage polarization," Arteriosclerosis, Thrombosis, and Vascular Biology, vol. 33, no. 2, pp. 170-177, 2013.

[128] D. Zhou, C. Huang, Z. Lin et al., "Macrophage polarization and function with emphasis on the evolving roles of coordinated regulation of cellular signaling pathways," Cellular Signalling, vol. 26, no. 2, pp. 192-197, 2014.

[129] D. Rückerl, S. J. Jenkins, N. N. Laqtom et al., "Induction of IL4Ralpha-dependent microRNAs identifies PI3K/Akt signaling as essential for IL-4-driven murine macrophage proliferation in vivo," Blood, vol. 120, no. 11, pp. 2307-2316, 2012.

[130] X. Jiang, M. Xue, Z. Fu et al., "Insight into the effects of adipose tissue inflammation factors on miR-378 expression and the underlying mechanism," Cellular Physiology and Biochemistry, vol. 33, no. 6, pp. 1778-1788, 2014.

[131] T. Kagiya and S. Nakamura, "Expression profiling of microRNAs in RAW264.7 cells treated with a combination of tumor necrosis factor alpha and RANKL during osteoclast differentiation," Journal of Periodontal Research, vol. 48, no. 3, pp. 373-385, 2013.

[132] B. Ell, L. Mercatali, T. Ibrahim et al., "Tumor-induced osteoclast miRNA changes as regulators and biomarkers of osteolytic bone metastasis," Cancer Cell, vol. 24, no. 4, pp. 542-556, 2013. 


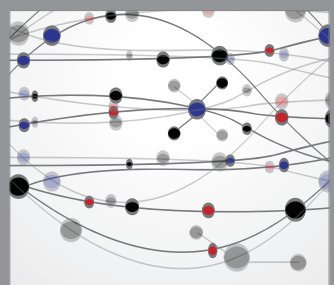

The Scientific World Journal
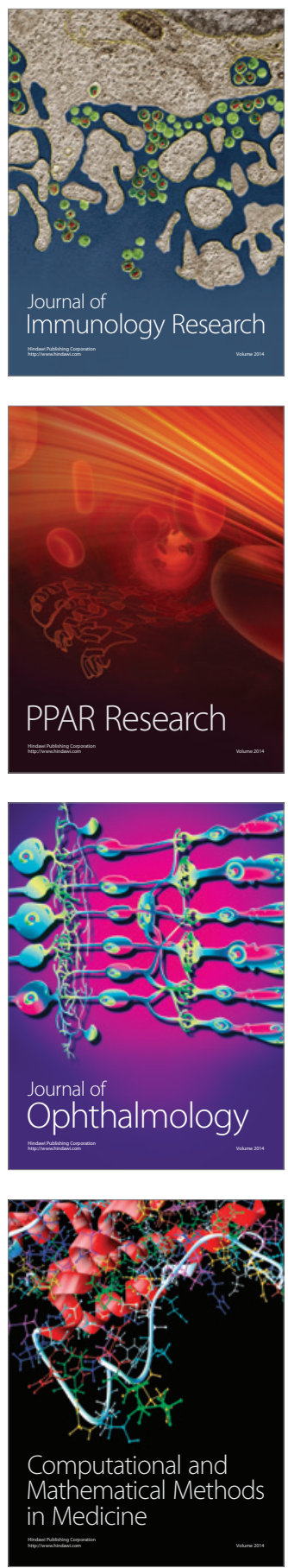

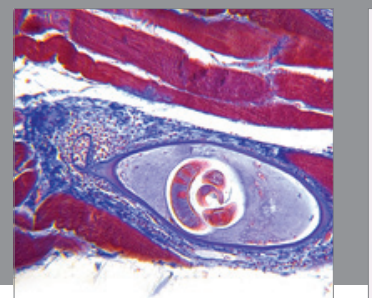

Gastroenterology

Research and Practice
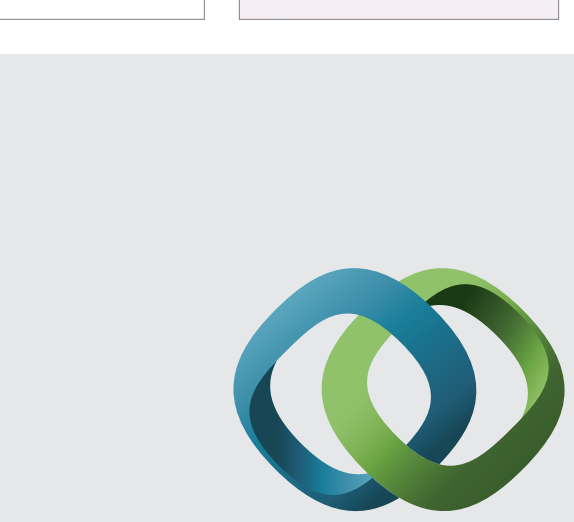

\section{Hindawi}

Submit your manuscripts at

http://www.hindawi.com
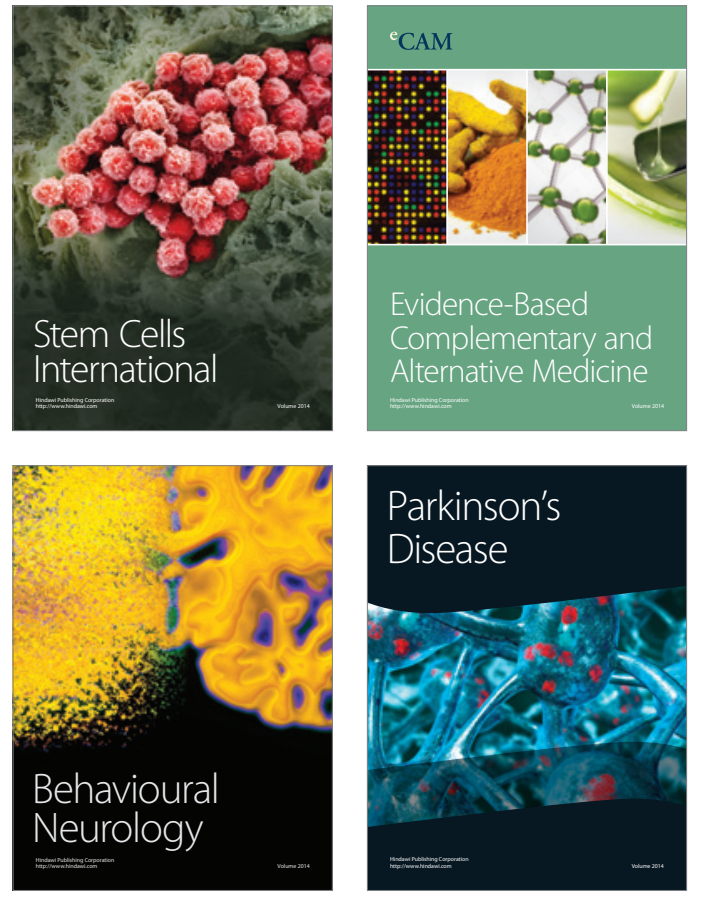
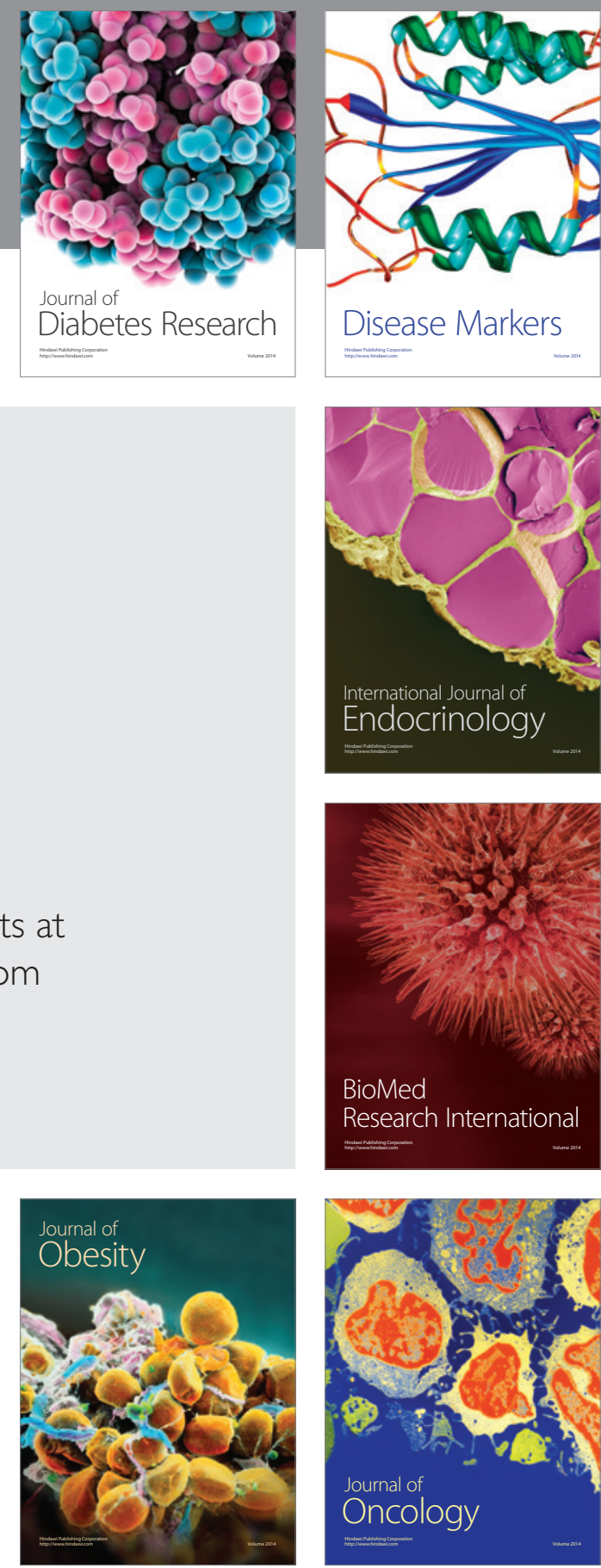

Disease Markers
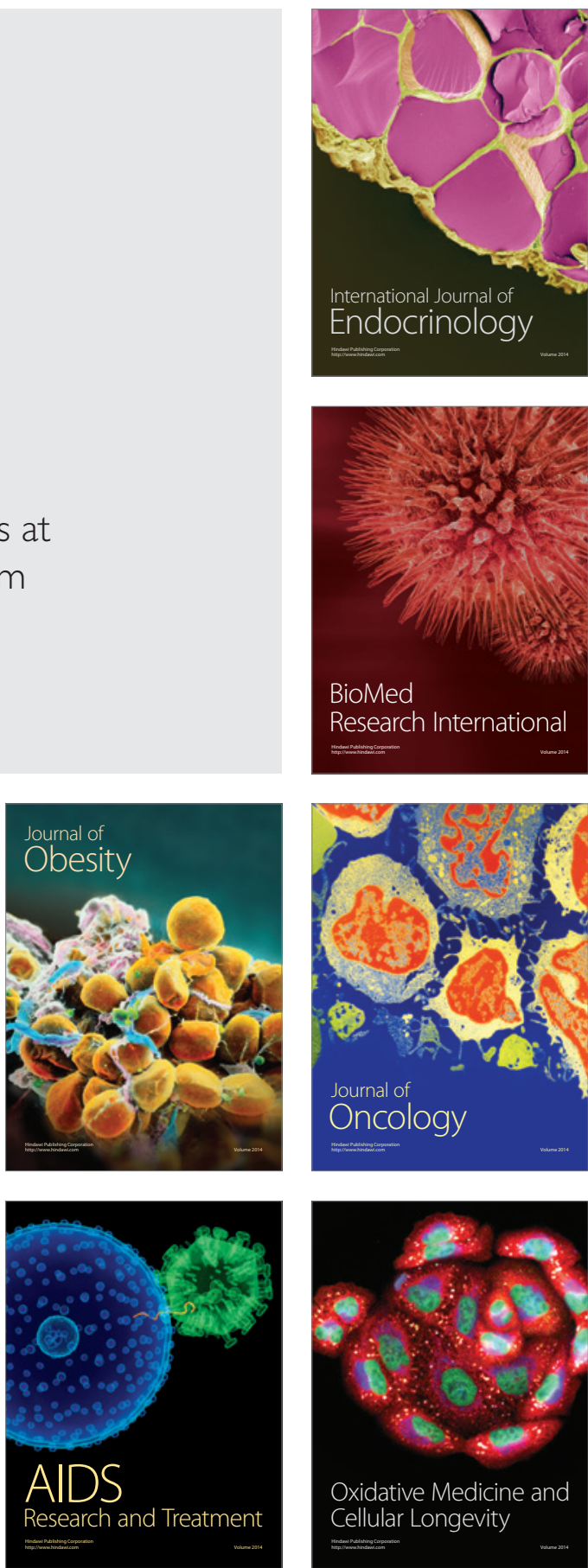